\title{
EXTRAÇÃO SEMIAUTOMÁTICA DE RODOVIAS NO ESPAÇO-OBJETO: USO INTEGRADO DE UM ESTEREOPAR DE IMAGENS AÉREAS E UM MDT
}

\author{
Semiautomatic road extraction in the object-space: integrated use of a stereopair of \\ aerial images and a DTM \\ ÉRICO FERNANDO DE OLIVEIRA MARTINS ${ }^{1,2}$ \\ ALUIR PORFÍRIO DAL POZ ${ }^{2}$ \\ RODRIGO BRUNO ZANIN ${ }^{1,2}$ \\ ${ }^{1,2}$ Universidade do Estado de Mato Grosso - Unemat \\ Faculdade de Ciências Exatas - FACIEX \\ Departamento de Matemática \\ profericomartins@unemat-net.br; rodrigo.zanin@unemat-net.br \\ ${ }^{2}$ Universidade Estadual Paulista - Unesp \\ Faculdade de Ciências e Tecnologia - FCT \\ Programa de Pós-Graduação em Ciências Cartográfica \\ Departamento de Cartografia \\ aluir@fct.unesp.br
}

\begin{abstract}
RESUMO
Neste artigo é proposto um método semiautomático para extração de rodovias combinando um estereopar de imagens aéreas de baixa resolução com um poliedro gerado a partir de um modelo digital do terreno (MDT). O problema é formulado no espaço-objeto através de uma função objetivo que modela o objeto 'rodovia' como uma curva suave e pertencente a uma superfície poliédrica. A função objetivo proposta depende também de informações radiométricas, que são acessadas no espaço-imagem via relação de colinearidade entre pontos da rodovia no espaçoobjeto e os correspondentes nos espaços imagem do estereopar. A linha poligonal que melhor modela a rodovia selecionada é obtida por otimização no espaço-objeto da função objetivo, tendo por base o algoritmo de programação dinâmica. $\mathrm{O}$ processo de otimização é iterativo e dependente do fornecimento por um operador de uma aproximação inicial para a rodovia selecionada. Os resultados obtidos mostraram que o método é robusto frente a anomalias existentes ao longo das rodovias, tais como obstruções causadas por sombras e árvores.
\end{abstract}


Palavras-chave: Programação Dinâmica; Modelo de Rodovia; Extração de Feição; Extração de Rodovia; Imagens Aéreas.

\section{ABSTRACT}

In this paper a semiautomatic method is proposed for road extraction by combining a stereoscopic pair of low-resolution aerial images and a polyhedron generated by using a digital terrain model (DTM). The problem is formulated in the object space by means of an objective function which models the object 'road' as a smooth curve that belongs to a polyhedral surface. The proposed objective function also depends on the radiometric information, which is accessed in the image space via collinearity relation between road points, in the object space and corresponding points in the image spaces of stereoscopic images. The polyline providing the best representation for a selected road is obtained in the object space via optimization of the objective function by using the dynamic programming algorithm. The optimization process is iterative and an operator needs to supply an initial polyline approximating the selected road. The obtained results showed that the method is robust against anomalies existing along roads, such as obstruction caused by shadows and trees.

Keywords: Dynamic Programming; Road Model; Feature Extraction; Road Extraction; Aerial Images.

\section{INTRODUÇÃO}

Os métodos para a extração de rodovias em imagens aéreas e de satélite são importantes no contexto de captura e atualização de informações espaciais. Diversas pesquisas nesta área têm sido realizadas desde a década de 1970, podendo se citar os trabalhos pioneiros de Bajcsy e Tavakoli (1976) e Quam (1978).

A maioria dos estudos realizados envolve inúmeros modelos e estratégias formulados no espaço-imagem. As estratégias semiautomáticas podem geralmente ser categorizadas como seguidores de rodovia (McKEOWN e DENLINGER, 1988, KIM et al., 2004, ZHOU et al., 2006, LIN et al., 2008), modeladores baseados em splines (HU et al., 2004), otimizadores baseados em snakes (KASS et al., 1987, NEUENSCHWADER et al., 1997, AGOURIS et al., 2000, YANG e KE-JU, 2009, GÖPFERT et al., 2011) e em programação dinâmica (PD) (GRÜEN e Li, 1997, MERLET e ZERUBIA, 1996, DAL POZ et al., 2010a, 2010b). Por outro lado, os métodos automáticos requerem grande sofisticação na integração de informação contextual e conhecimento a priori em modelos e estratégias para a extração de rodovia (BAUMGARTNER et al., 1999; HU et al., 2007, POULLIS e YOU, 2010, KIRTHIKA e MOOKAMBIGA, 2011). Detalhes adicionais sobre o estado da arte envolvendo métodos para a extração de rodovias no espaço-imagem podem ser encontrados em Yibo (2009).

Poucos métodos têm sido desenvolvidos para extrair rodovias diretamente no espaço-objeto. Estes métodos podem basear-se em uma única imagem (modo mono) combinada com um modelo digital do terreno (MDT) ou em duas ou mais imagens 
(modo estéreo) de um mesmo sensor ou de diferentes sensores. O modo estéreo pode tirar vantagem da integração de um MDT no processo de extração, o que potencialmente pode tornar o processo de extração mais estável. Em ambos os modos, o tratamento de oclusões e falsas hipóteses de rodovia pode ser realizada de forma mais eficiente (GRUEN e LI, 1997, HINZ et al., 2001, HINZ e WIEDEMANN, 2004). Uma vantagem relevante de delinear rodovias no espaçoobjeto é que a restrição de suavidade vertical do eixo de rodovia pode ser facilmente introduzida no processo de extração. Este tipo de restrição não pode ser forçado nos modelos e estratégias formuladas no espaço-imagem. Provavelmente o primeiro método formulado no espaço-objeto foi o LSB-Snakes (Least-Squares B-spline Snakes) (GRUEN e LI, 1997), que combina snakes e B-splines para extrair rodovias em 3D a partir de múltiplas imagens aéreas. Hinz e Baumgartner (2003) propuseram um sistema híbrido para a extração automática de rodovias a partir de um estereopar de imagens aéreas de alta resolução, em que segmentos de rodovia são primeiramente extraídos no espaço-imagem e então são projetados no espaço-objeto para a reconstrução completa da malha viária. Zhang (2004) propôs outro sistema híbrido para a extração em $3 \mathrm{D}$ de rodovias usando um estereopar de imagens coloridas, tendo por referência rodovias existentes numa base de dados. Dal Poz et al. (2010a) associaram uma imagem aérea de alta resolução com um MDT para extrair rodovias diretamente no espaço-objeto. Este método foi estendido em Dal Poz et al. (2010b), passando o modelo de rodovia a depender apenas de um estereopar de imagens aéreas de alta resolução. Em ambos os casos o algoritmo de PD foi utilizado para otimizar os respectivos modelos de rodovia.

Neste artigo é proposto um método semiautomático para extração de rodovias no espaço-objeto combinando um estereopar de imagens aéreas de baixa resolução com um poliedro gerado a partir de um MDT. Este método trata adequadamente rodovias que se manifestam no estereopar como feições lineares. Na prática, uma rodovia pode ser modelada como uma feição linear quando sua largura na imagem varia no intervalo 1-3 pixels. Nesse caso, a imagem envolvida é classificada como de baixa resolução no contexto de métodos de extração de rodovia (BAUMGARTNER et al., 1999). O método proposto é uma extensão do método proposto em Dal Poz et al. (2010b). O diferencial básico da presente proposta consiste na introdução de uma restrição geométrica, pela qual a linha poligonal extraída deve pertencer à superfície de um poliedro gerada a partir de um MDT em estrutura triangular (TIN). Esta restrição permite tratar melhor as obstruções presentes no leito da rodovia, como as causadas por árvores. Este artigo está organizado em quatro seções principais. A Seção 2 apresenta os detalhes relevantes do método proposto. Os principais resultados obtidos e as respectivas análises são apresentados na Seção 3. O artigo é finalizado na Seção 4, onde são apresentadas as principais conclusões e perspectivas futuras. 


\section{MÉTODO}

O método proposto baseia-se num modelo de rodovia formulado no espaçoobjeto a partir de um estereopar de imagens aéreas, proposto em Dal Poz et al. (2010b). Apresenta-se a seguir (Subseção 2.1) uma breve descrição deste modelo, sendo que maiores detalhes podem ser encontrados na mencionada referência. A subseção 2.2 apresenta as modificações no modelo de rodovia proposto em Dal Poz et al. (2010b) e também na forma de amostragem do espaço de busca.

\subsection{Modelo de Rodovia para um Estereopar de Imagens Aéreas}

Assumindo-se que uma rodovia numa imagem de baixa resolução possa ser representada pela linha poligonal $P^{i}=\left\{p_{1}, \ldots, p_{n}\right\}$, onde $p_{i}$ é seu i-ésimo vértice, pode-se modelá-la matematicamente através da função objetivo (Equação 1) e da injunção de desigualdade (Equação 2), como segue (GRUEN e LI, 1997),

$$
\begin{gathered}
E=\sum_{i=1}^{n-1}\left(\left[E_{p 1}-\beta E_{p 2}+\gamma E_{p 3}\right] \times\left(1+\cos \left(\alpha_{1}-\alpha_{i+1}\right)\right) /\left|\Delta_{s_{i}}\right|\right) \\
C_{i}=\alpha_{i}-\alpha_{i+1}<T
\end{gathered}
$$

onde, $E_{p l}$ é uma função que depende do vértice $p_{i}$ e expressa o fato de que os pixels de rodovia são mais claros que seus vizinhos em ambas as margens da rodovia; $E_{p 2}$ é uma função que depende de dois pontos consecutivos $\left(p_{i-1}\right.$ e $\left.p_{i}\right)$ da linha poligonal $P^{i}$ e expressa o fato de que os níveis de cinza ou cor da rodovia não variam muito, sobretudo em curtas distâncias; $E_{p 3}$ é uma função que depende do vértice $p_{i}$ e expressa o fato de que uma rodovia é uma feição linear de alto valor de brilho; $\alpha_{\mathrm{i}}$ é a direção do segmento de reta definido pelos pontos $p_{i-1}$ e $p_{i} ; \beta$ e $\gamma$ são constantes positivas; $\left|\Delta_{\mathrm{si}}\right|$ é a distância entre os pontos $p_{i-1}$ e $p_{i}$; e $T$ é um limiar angular que limita a mudança de direção entre dois segmentos sucessivos da linha poligonal $P^{i}$.

A Equação 1 mostra que somente três pontos consecutivos $\left(p_{i-1}, p_{i}, p_{i+1}\right)$ da linha poligonal $P^{i}$ são simultaneamente inter-relacionados e que, em decorrência, pode ser decomposta numa soma de $n-1$ subfunções do tipo $E_{\mathrm{i}}\left(p_{i-1}, p_{i}, p_{i+1}\right)$, conforme mostra a Equação 3.

$$
E=\sum_{i=1}^{n-1} E_{i}\left(p_{i-1}, p_{i}, p_{i+1}\right)
$$

A solução deste problema é uma linha poligonal $P^{i}=\left\{p_{l}, \ldots, p_{n}\right\}$ que representa uma rodovia e corresponde ao máximo da função objetivo dada pela Equação 3. Esta função objetivo pode ser colocada em função de coordenadas de vértices de pontos da correspondente linha poligonal num referencial do espaço-objeto. Para tal, basta estabelecer, via equações de colinearidade, as relações matemáticas entre as coordenadas linha $\left(L_{i}\right)$ e coluna $\left(C_{i}\right)$ do vértice $p_{i}$ com as coordenadas do ponto $P_{i}$ no 
espaço-objeto. Sendo $P_{i}$ com coordenadas $E_{i}$ e $N_{i}$ do sistema UTM (Universe Transverse Mercator) e com altura elipsoidal $h_{i}$, demonstra-se que a equação 3 pode ser colocada na forma,

$$
E=\sum_{i=1}^{n-1} E_{i}\left(P_{i-1}\left(E_{i-1}, N_{i-1}, h_{i-1}\right), P_{i}\left(E_{i}, N_{i}, h_{i}\right), P_{i+1}\left(E_{i+1}, N_{i+1}, h_{i+1}\right)\right)
$$

A função objetivo dada pela Equação 4 é ambígua, visto que existem infinitas linhas poligonais no espaço-objeto que se projetam no espaço-imagem numa mesma linha poligonal. A fim de remover esta ambiguidade e possibilitar a obtenção de solução única, Dal Poz et al. (2010b) desenvolveram uma outra função objetivo para um estereopar, dada pela soma das funções objetivo para as imagens esquerda e direita, ambas de baixa resolução, ficando,

$$
\mathrm{E}^{T}=E^{l}+E^{r}=\sum_{\mathrm{i}=1}^{\mathrm{n}-1} \mathrm{E}_{\mathrm{i}}^{T}\left(P_{i-1}\left(E_{i-1}, N_{i-1}, h_{i-1}\right), P_{i}\left(E_{i}, N_{i}, h_{i}\right), P_{i+1}\left(E_{i+1}, N_{i+1}, h_{i+1}\right)\right)
$$

onde, $E^{l}$ é a função objetivo (Equação 4) envolvendo a rodovia R no espaço-objeto e a rodovia $r$ na imagem esquerda; $E^{r}$ é a função objetivo (Equação 4) envolvendo o eixo da rodovia $R$ e o eixo da rodovia $r$ na imagem direita; e $\mathrm{E}_{\mathrm{i}}^{T}$ resulta do agrupamento de termos semelhantes de $E^{l}$ e $E^{r}$.

Figura 1 - Princípio do modelo de rodovia para um estereopar de imagens.

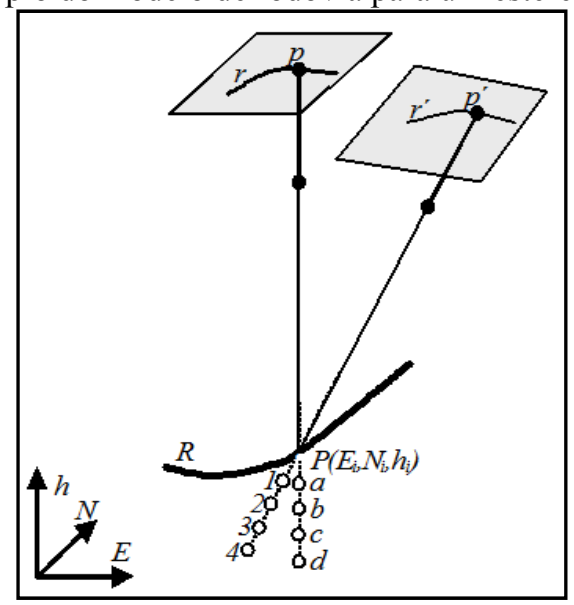

A Equação 5 pode ser teoricamente justificada com base na Figura 1. Seja $P^{o}=$ $\left\{P_{l}\left(E_{l}, N_{l}, h_{l}\right) ; \ldots ; P_{i}\left(E_{i}, N_{i}, h_{i}\right) ; \ldots ; P_{n}\left(E_{n}, N_{n}, h_{n}\right)\right\}$ a linha poligonal que descreve Bol. Ciênc. Geod., sec. Artigos, Curitiba, v. 18, nº 3, p.446-463, jul-set, 2012. 
corretamente o eixo da rodovia $R$. Então, $P^{o}$ maximiza ambas as funções objetivo $E^{l}$ e $E^{\mathrm{r}}$. A função objetivo $E^{l}$ assume o mesmo máximo se o ponto $P_{i}\left(E_{i}, N_{i}, h_{i}\right)$ for substituído pelos pontos $a, b, c$, ou $d$, mas a função objetivo $E^{\mathrm{r}}$ seria maximizada apenas pela linha poligonal $P^{o}$. O inverso ocorreria se o ponto $P_{i}\left(E_{i}, N_{i}, h_{i}\right)$ fosse substituído pelos pontos $1,2,3$, ou 4 . Portanto, somente a linha poligonal $P^{o}$ maximize simultaneamente as funções objetivo $E^{l}$ e $E^{\mathrm{r}}$ e, como são funções positivas, o mesmo ocorre com a função objetivo dada pela Equação 5.

Outra forma de definição da função objetivo é através do produto entre $E^{l}$ e $E^{\mathrm{r}}$, mas a equação resultante não poderia ser decomposta em subfunções dependentes simultaneamente de poucas variáveis, que é essencial para a solução do problema de otimização por PD, que é o caso da Equação 5, onde se constata que apenas nove variáveis se inter-relacionam simultaneamente. Refere-se na sequência à Equação 5 como modelo estéreo de rodovia.

\subsection{Modelo Estéreo de Rodovia Modificado e Esquema de Otimização}

O processo de extração inicia com uma linha poligonal definida no espaçoobjeto por alguns pontos sementes, que devem ser fornecidos por um operador de forma esparsa ao longo da rodovia a ser extraída. Como uma regra geral, poucos pontos semente são necessários ao longo de trechos de curva suave, ocorrendo o oposto em trechos de curva sinuosa. Dada a necessidade de o operador visualizar a rodovia para fornecer os pontos sementes, a estratégia adotada é medi-los numa das imagens do estereopar e projetá-los sobre o MDT via algoritmo de monorrestituição (MAKAROVIK, 1973).

Nas etapas seguintes a linha poligonal inicial (Figura 2a) é progressivamente adensada e refinada no espaço-objeto, em ciclos iterativos de otimização, até descrever adequadamente o eixo da rodovia. A densificação é realizada via interpolação linear de pontos centrais entre cada par de vértices preexistentes (Figura 2b).

Figura 2 - (a) Linha poligonal inicial com três pontos sementes. (b) Adensamento.

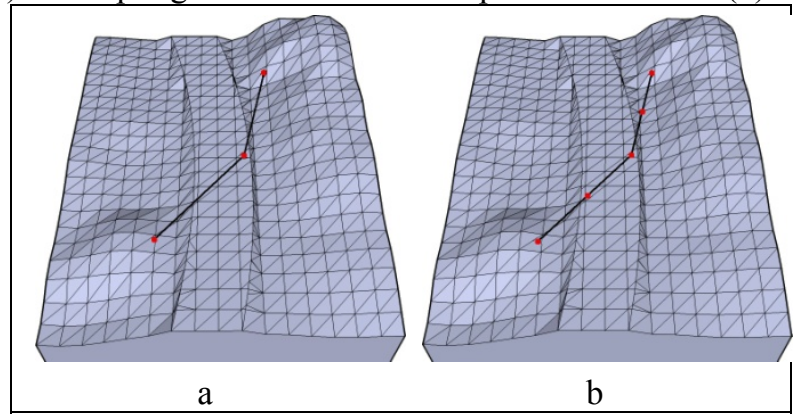


Cada vértice da linha poligonal adensada serve de referência para amostrar vértices candidatos ao vértice ótimo. Se para cada um dos $n$ vértices da linha poligonal adensada forem amostrados $m$ vértices candidatos, haverá no espaço de busca $m^{n}$ linhas poligonais candidatas na iteração atual. Portanto, é recomendável amostrar o menor número possível de candidatas em cada vértice. Para minimizar o número de vértices candidatos, o espaço de busca é limitado a uma linha poligonal denominada linha poligonal de busca. As linhas poligonais de busca são obtidas pela intersecção entre o poliedro gerado a partir do MDT e planos perpendiculares à linha poligonal adensada (Figura 3a,b). Os vértices candidatos são amostrados ao longo das linhas poligonais de busca, de forma simétrica e regular a partir dos vértices da linha poligonal adensada (Figura 3c). A extensão das linhas poligonais de busca depende do quão próximo o operador forneceu os pontos sementes. Entretanto, para maximizar o raio de convergência e garantir um resultado acurado, adota-se uma estratégia multi-resolução. Nas primeiras iterações são adotadas linhas poligonais de busca de resolução menor, da ordem da largura de uma rodovia, garantindo uma maior região de busca e, nas iterações seguintes, deve-se adotar maior resolução (da ordem de $1 / 3$ da largura da rodovia), permitindo um posicionamento acurado da linha poligonal ótima sobre a rodovia.

Figura 3 - (a) Planos perpendiculares à linha poligonal adensada. (b) Linhas poligonais de busca definidas por intersecção dos planos com o MDT. (c) Linhas poligonais de busca amostradas.

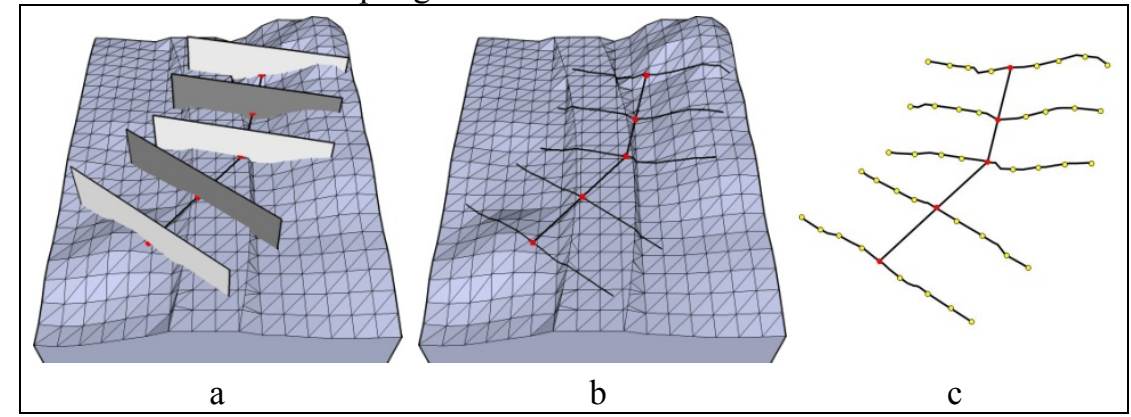

A Figura 3c mostra um exemplo com cinco linhas poligonais de busca, cada qual com oito vértices candidatos amostrados. A amostragem desses pontos é feita através da equação da reta na forma paramétrica, que é formulada para cada segmento de reta da linha poligonal de busca. Cada ponto $P_{i}\left(E_{i}, N_{i}, h_{i}\right)$ é calculado em função do parâmetro $t_{i}$ da reta, que é a distância ao longo da linha poligonal de busca entre o ponto central (vértice da linha poligonal de busca) e o ponto a ser amostrado $\left(P_{i}\left(E_{i}, N_{i}, h_{i}\right)\right)$. Portanto, cada ponto $P_{i}\left(E_{i}, N_{i}, h_{i}\right)$ depende apenas da distância $t_{i}$, permitindo escrever a Equação 5 na forma, 


$$
E^{T}=\sum_{i=1}^{n-1} E_{i}^{T}\left(P_{i-1}\left(t_{i-1}\right), P_{i}\left(t_{i}\right), P_{i+1}\left(t_{i+1}\right)\right)
$$

A Equação 6 mostra que apenas três variáveis $\left(t_{i-1}, t_{i}, t_{i+1}\right)$ estão interrelacionadas simultaneamente, em vez de nove da função objetivo (Equação 5) proposta em Dal Poz et al. (2010b). Vale ressaltar que a Equação 6 não é apenas uma parametrização da Equação 5 para possibilitar a redução de variáveis simultaneamente inter-relacionadas. Permite também, diferente da Equação 5, extrair a linha poligonal que representa uma dada rodovia de forma que seus vértices pertençam a uma estrutura poliédrica. A Equação 6 permite que o problema seja formulado de maneira a ser eficientemente otimizado via PD, visto que a condição básica (isto é, que poucas variáveis estejam inter-relacionadas simultaneamente) para aplicação deste algoritmo de otimização é plenamente atendida. Detalhes sobre o algoritmo de PD podem ser encontrados na literatura clássica de visão computacional (por exemplo, BALLARD e BROWN, 1982) ou em teses e dissertações (por exemplo, GALLIS, 2006, MARTINS, 2010).

A Figura 4 exemplifica de forma simples a evolução do processo de refinamento da linha poligonal inicial adensada. A Figura 4a mostra que, após a iteração zero (primeiro ciclo de otimização), que compreende um adensamento e uma otimização por PD, a linha poligonal adensada (linha contínua) será substituída por uma nova linha poligonal (linha tracejada). Esta linha poligonal é novamente adensada e novas linhas poligonais de busca são criadas (Figura 4b). As iterações prosseguem até que um critério de parada seja atendido, tendo como resultado final um conjunto de pontos que descrevem o eixo da rodovia no espaço-objeto (Figura $4 c)$. O resultado de cada iteração é o vetor ótimo $\left(t_{o}, \ldots, t_{n}\right)$, a partir do qual são calculadas as coordenadas dos vértices da linha poligonal ótima.

Figura 4 - (a) Eixo de referência resultante da iteração zero. (b) Linhas de busca do eixo de referência. (c) Sobreposição do eixo final com o MDT.

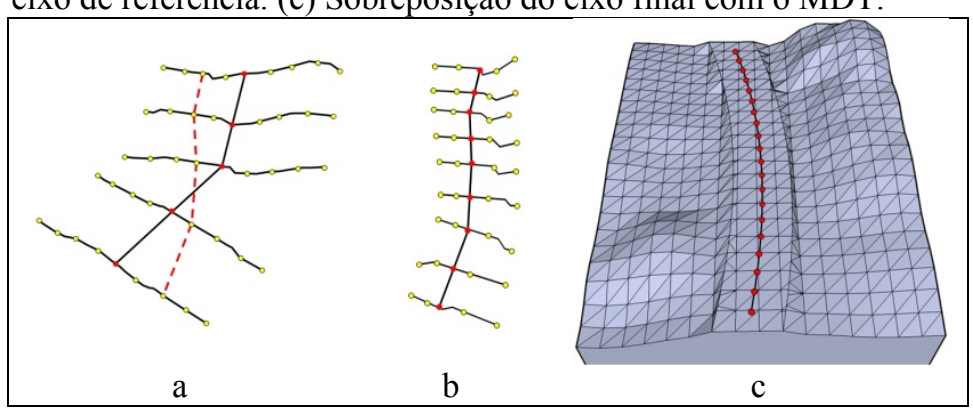

Foram utilizados dois critérios de parada. O primeiro estabelece que a média das distâncias $\left(\ldots, d_{j}, d_{j+1}, \ldots\right)$ entre os vértices da linha poligonal obtida no final de 
cada iteração deve ser inferior a $1 \mathrm{~m}$ (Figura 5a), evitando que os vértices sejam demasiadamente agrupados. O segundo critério define que entre uma iteração e a seguinte, o deslocamento médio dos vértices $\left(\ldots, \mathrm{d}_{\mathrm{k}}, \mathrm{d}_{\mathrm{k}+1}, \mathrm{~d}_{\mathrm{k}+2}, \ldots\right)$ que formam $\mathrm{o}$ eixo de referência deve ser inferior ao limiar de $0,2 \mathrm{~m}$ (Figura $5 \mathrm{~b}$ ).

Figura 5 - (a) Primeiro critério de parada. (b) Segundo critério de parada.

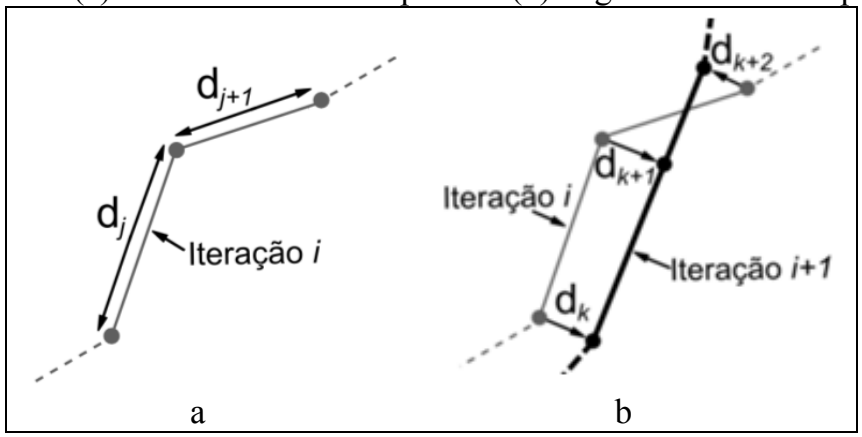

No decorrer de cada iteração, as linhas poligonais candidatas não suaves são descartadas do processo de otimização por PD. Isso é plausível porque as rodovias são caracterizadas como faixas suaves. O critério de identificação de linhas poligonais não suaves baseia-se na análise dos ângulos de deflexão horizontal e vertical. Uma linha poligonal é considerada como suave se ambos os ângulos de deflexão estão abaixo de um limiar (tipicamente de $5^{\circ}$ ) em todos seus vértices.

\section{EXPERIMENTOS E ANÁLISE DOS RESULTADOS}

Para avaliar o método proposto, foram realizados experimentos com dois estereopares de imagens aéreas de uma região da Suíça que integram um projeto demonstrativo do sistema fotogramétrico Leica Photogrammetry Suíte (LPS). Estas imagens originalmente possuíam 9286 x 9496 pixels e GSD (Ground Sampling Distance) da ordem de $0,2 \mathrm{~m}$. Como a metodologia proposta é formulada para imagens de baixa resolução, as imagens originais foram reamostradas de modo a gerar imagens com 1547 x 1582 pixels e GSD de 1,3 m. A rigor, imagens com esse GSD são consideradas, no contexto de métodos para a extração de rodovias, como de média resolução. De acordo com Baumgartner et al. (1999), imagens de baixa resolução necessitam ter GSD maior que $2 \mathrm{~m}$, equivalendo a rodovias com larguras variando no intervalo aproximado de 1-3 pixels. Esta é a faixa ideal de variação da largura de rodovia na imagem para a aplicação do método proposto. Nas imagens reamostradas as rodovias variam em largura de 2 a 6 pixels. Isto permitirá testar o método em situações que o mesmo pode perder a eficiência. O MDT utilizado nos experimentos foi gerado no LPS em estrutura TIN a partir das imagens originais. A resolução do MDT deve possibilitar a diferenciação entre textura (fina) do leito de 
uma rodovia e a textura (grosseira) das regiões adjacentes à rodovia. A resolução média do MDT gerado foi de $1 \mathrm{~m}$.

Os resultados obtidos foram avaliados visual e numericamente. A avaliação visual consistiu em analisar as linhas poligonais extraídas e superpostas numa das imagens do estereopar. A avaliação numérica foi realizada por meio dos seguintes parâmetros de qualidade: Completeza e Correção (WIEDEMANN e HINZ, 1999; MAIA, 2003), além do Erro Médio Quadrático (RMS) (GEMAEL, 1994). A completeza é conceitualmente a porcentagem do que foi extraído corretamente em relação ao que deveria ser extraído (eixo verdadeiro). Enquanto a correção é a porcentagem do que foi extraído corretamente em relação ao total que foi extraído. Já o Erro Médio Quadrático (RMS) permite inferir sobre a acurácia da metodologia de extração. A fim de determinar os índices de completeza, correção e RMS, foi necessário prover dados de referência através da extração manual das rodovias no LPS. O índice de completeza não é muito significativo para avaliar o método proposto, visto que sempre algum resultado é gerado para, pelo menos, realizar uma descrição grosseira do eixo da rodovia. Fica a cargo do índice de correção a verificação de quanto está correto. Uma rodovia, ou parte dela, é considerada correta se as distâncias entre seus vértices e a respectiva rodovia de referência são menores ou iguais a semi-largura da rodovia considerada.

Foram extraídos, pelo método proposto, 40 trechos de rodovia. No primeiro estereopar (Figura 6a), foram realizadas 26 extrações com comprimento variando de 150 a $2.200 \mathrm{~m}$, totalizando $12.705 \mathrm{~m}$ de rodovias (Figura 6b). No segundo estereopar (Figura 7a), foram realizadas 9 extrações com trechos variando de 130 a $1.108 \mathrm{~m}$, totalizando neste estereopar $5.037 \mathrm{~m}$ de rodovias extraídas (Figura 7b).

Figura 6 - (a) Imagem esquerda do estereopar. (b) Malha extraída.

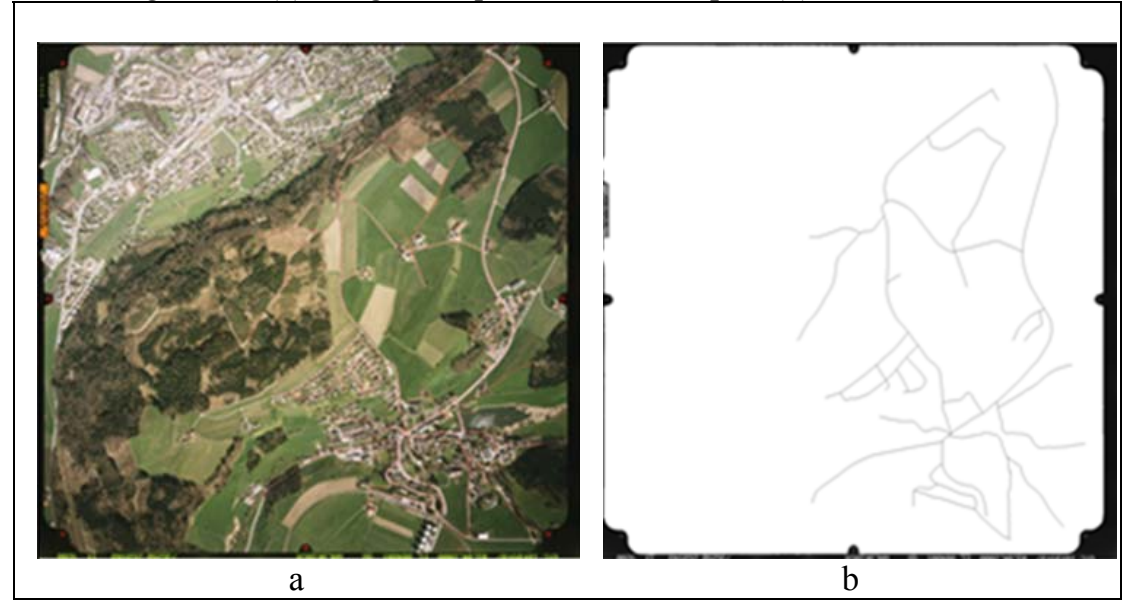


Figura 7 - (a) Imagem esquerda do estereopar. (b) Malha extraída.

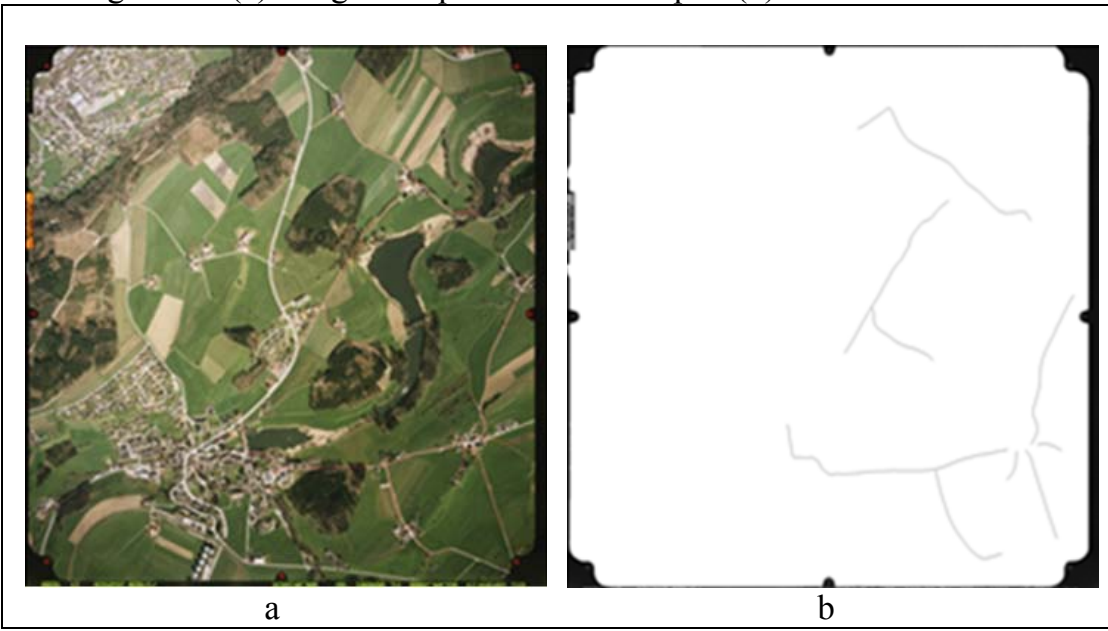

A fim de avaliar o desempenho do método, serão analisados a seguir os resultados obtidos para quatro segmentos de rodovias. Estes trechos de rodovia apresentam condições diferentes de contraste, suavidade, sinuosidade e de espessura. Poderá também ser verificada ao longo destes segmentos a presença de diferentes anomalias, tais como obstruções perspectivas e por sombra. As principais características das extrações selecionadas são apresentadas na Tabela 1. Em especial, as larguras das rodovias foram determinadas diretamente sobre o TIN usando um aplicativo gráfico.

Tabela 1 - Resumo das extrações selecionadas.

\begin{tabular}{c|c|c|c|c|c}
\hline Trecho & $\begin{array}{c}\text { Extensão } \\
\text { extraída }(\mathrm{m})\end{array}$ & $\begin{array}{c}\text { Pontos } \\
\text { Sementes }\end{array}$ & Iterações & $\begin{array}{c}\text { Pontos } \\
\text { Extraídos }\end{array}$ & $\begin{array}{c}\text { Largura da } \\
\text { Rodovia (m) }\end{array}$ \\
\hline 1 & 504 & 3 & 5 & 31 & 3,75 \\
\hline 2 & 655 & 3 & 6 & 63 & 2,21 \\
\hline 3 & 672 & 4 & 5 & 22 & 5,08 \\
\hline 4 & 924 & 6 & 5 & 79 & 3,31 \\
\hline
\end{tabular}

O trecho 1 de rodovia (Figura 8a) apresenta bom contraste com as regiões adjacentes, apresentando também uma geometria bem suave. A Figura $8 \mathrm{~b}$ mostra que, embora existam algumas anomalias que provocam a mudança de largura e desaparecimento da borda da rodovia, o resultado é aceitável. 
Figura 8 - (a) Pontos sementes. (b) Eixo extraído do trecho 1.

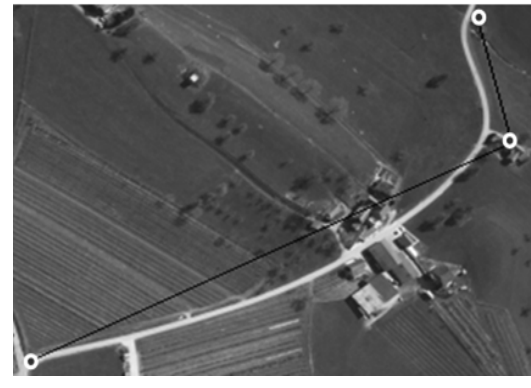

$\mathrm{a}$

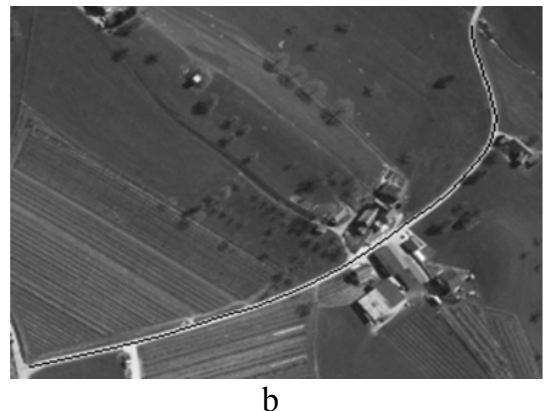

b

No exemplo envolvendo o trecho 2 de rodovia (Figura 9a) constam elementos de contexto que impõem maior dificuldade ao método. O retângulo maior destaca uma área de solo exposto que prejudica o contraste da rodovia com uma das regiões adjacentes. O retângulo menor destaca uma área suburbana com construções e cruzamento de vias. O resultado obtido (Figura 9b) comprova a robustez do método, mesmo com o uso de apenas três pontos sementes (Figura 9a).

Figura 9 - (a) Pontos sementes. (b) Eixo extraído do trecho 2.

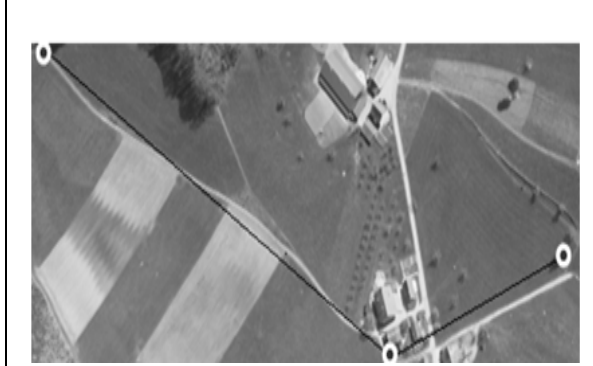

a

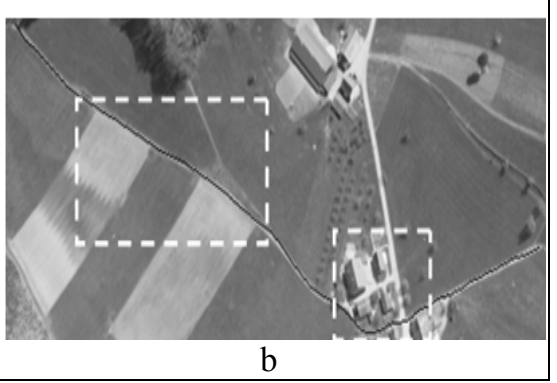

b

O trecho 3 de rodovia (Figura 10a) descreve uma curva prolongada, com duas regiões de oclusões. Na Figura 10b, o retângulo menor destaca edificações e sombras projetadas sobre a rodovia e o retângulo maior destaca uma área de bosque que praticamente obstrui um longo segmento da rodovia. Foram utilizados quatro pontos sementes, sendo dois bem próximos para facilitar a captura do sinal da rodovia no trecho obstruído pelo bosque, que está aproximadamente compreendido entre os dois pontos sementes intermediários. Outra característica importante do método que auxilia na extração de segmentos parcialmente obstruídos e de curva suave é a restrição de suavidade incorporada no modelo de rodovia. O resultado da extração é satisfatório, embora parte do eixo extraído tangencie uma das bordas da 
rodovia. Isso ocorre devido à característica do método de modelar melhor rodovias com até três pixels de espessura, o que não é o caso do trecho em análise.

Figura 10 - (a) Pontos sementes. (b) Eixo extraído do trecho 3.

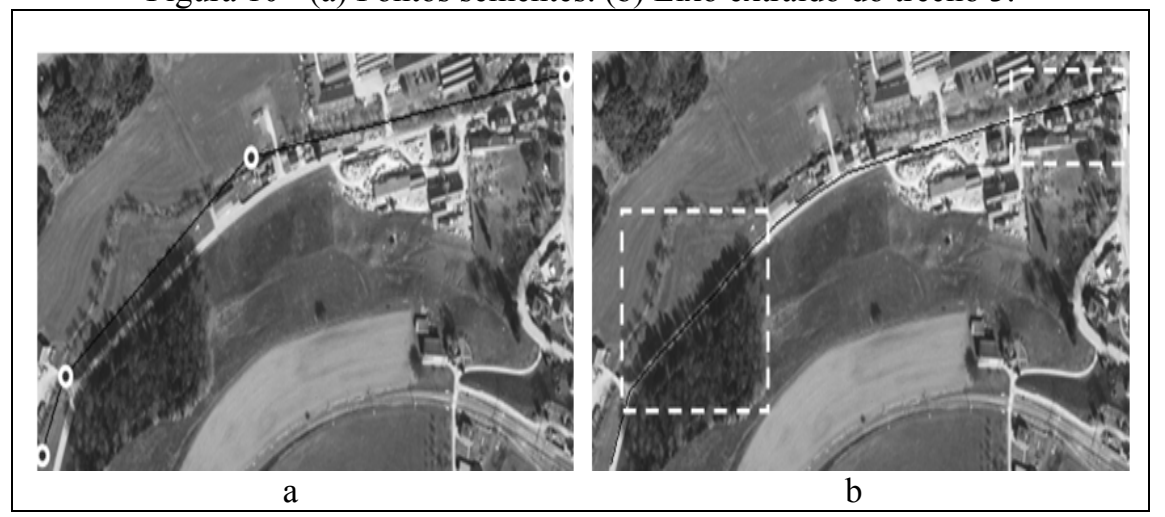

O trecho 4 de rodovia (Figura 11a) é relativamente complexo. Do ponto de vista geométrico, apresenta um segmento curto com curva sinuosa e outro longo e suave. Embora seja suave, este último segmento possui duas regiões obstruídas por vegetação e sombra, sendo que uma delas contém cruzamentos e edificações, conforme destacado pelo retângulo da Figura 11 b. Foram necessários seis pontos sementes para obter o resultado mostrado na Figura $11 \mathrm{~b}$ que, conforme mostra a Figura 11a, foram agrupados três a três para possibilitar a correta modelagem do segmento com curva sinuosa e do segmento parcialmente obstruído pela vegetação.

Figura 11 - (a) Pontos sementes. (b) Eixo extraído do trecho 4.

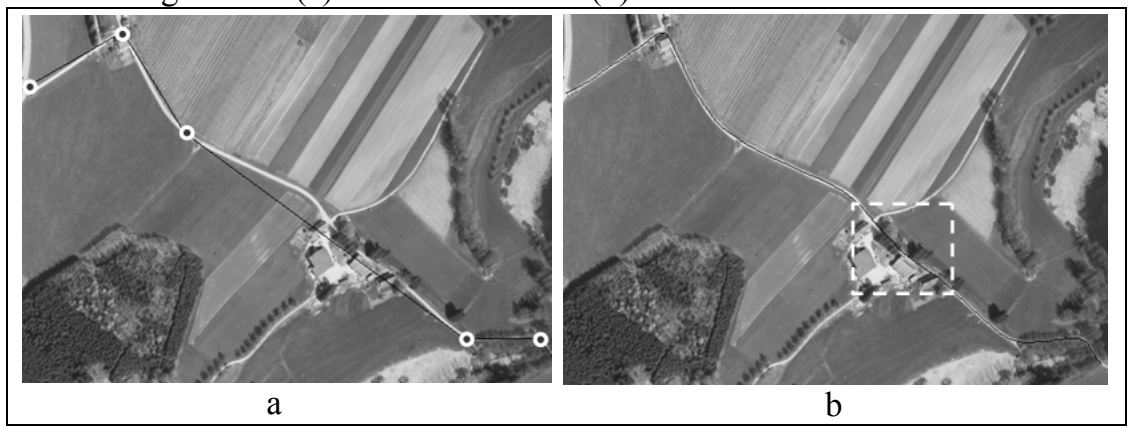

A Tabela 2 apresenta dados para subsidiar a análise numérica, de forma a respaldar as observações realizadas visualmente. 
Tabela 2 - Resultados da análise numérica dos trechos extraídos.

\begin{tabular}{c|c|c|c|c}
\hline Trecho & Figura & Completeza (\%) & Correção (\%) & RMS (metros) \\
\hline 1 & 08 & 100 & 83 & 2,10 \\
\hline 2 & 09 & 100 & 62 & 1,25 \\
\hline 3 & 10 & 100 & 77 & 2,12 \\
\hline 4 & 11 & 100 & 84 & 1,46 \\
\hline
\end{tabular}

Todos os trechos foram completamente extraídos, o que justifica, conforme mostra a Tabela 2, a obtenção do índice máximo (100\%) para a completeza. O pior resultado foi obtido na extração do trecho 2 de rodovia. Isso ocorreu porque a linha poligonal extraída tangenciou em várias partes uma das bordas da rodovia (Figura 9b). As regiões de rodovia margeadas com solo exposto e que atravessam a área suburbana, conforme destaques com retângulos na Figura 9b, contribuíram significativamente com o menor índice de correção. Já a extração do trecho 4 de rodovia apresentou o melhor índice de correção, embora seja o trecho de maior complexidade. Vale relembrar que a distribuição dos pontos sementes de forma estratégica nas proximidades das regiões críticas possibilitou a obtenção dos bons resultados mostrados na Figura $11 \mathrm{~b}$. Os demais trechos de rodovia (1 e 3) foram extraídos com índice de correção em torno de $80 \%$, o que pode ser considerado como satisfatório. O menor índice de correção entre ambos os trechos (62\%) foi obtido para o trecho 3 de rodovia, o que pode estar relacionado ao fato do respectivo trecho aparecer no estereopar com largura superior a três pixels. Isto contraria a hipótese básica assumida no desenvolvimento do modelo de rodovia, pelo qual a largura da rodovia deve situar entre 1 e 3 pixels. O índice RMS apresentou uma variação que acompanha a largura da rodovia. Para o trecho 2, com rodovia de 2,21 metros de largura, o RMS foi de 1,25 metros (0,96 pixel). Já para o trecho 3 , com rodovia de 5,08 metro de largura, o RMS atingiu 2,12 m (1,63 pixels). Os trechos 1 e 3 apresentaram os piores resultados em termos de RMS. Apesar destes trechos apresentarem geometria mais suave, os mesmos possuem larguras maiores, situação que se inverte para os trechos de melhores resultados (2 e 4). A variação do RMS em pixels indica que os resultados obtidos estão pouco dispersos em relação aos dados de referência, considerando se tratar de um processo que não conta com injunções de bordas.

Por último, apresenta-se um breve comparativo entre o método proposto neste artigo e os métodos propostos em Dal Poz et al. (2010a,b). Em Dal Poz et al. (2010a) foi combinado uma imagem aérea de altíssima resolução (GSD de aproximadamente $0,25 \mathrm{~m}$ ) com um MDT de $1 \mathrm{~m}$ de resolução. O RMS obtido para todo o conjunto de rodovias extraídas foi de $1,17 \mathrm{~m}$. A metodologia proposta em Dal Poz et al. (2010b) tinha por base um par estereoscópico envolvendo imagens aéreas com as mesmas características de resolução das que foram utilizadas para avaliar o método anterior. Um RMS global de $1,46 \mathrm{~m}$ foi obtido para todas as rodovias extraídas. Diferente dos dois métodos anteriores, que foram concebidos para o 
tratamento de imagens aéreas de diferentes resoluções, o presente método limita-se a modelagem de imagens de baixa resolução, motivo pelo qual foram utilizadas imagens com GSD de 1,3 m. A combinação de pares estereoscópicos envolvendo imagens com estas características com um MDT de $1 \mathrm{~m}$ de resolução possibilitou a obtenção de um RMS global na casa de 1,7 m. De uma forma geral, observa-se que: 1) entre os dois métodos anteriores, cujos dados de entrada provinham de mesma área teste, mostram claramente que o MDT influenciou positivamente na obtenção de um RMS melhor; 2) o RMS obtido com o método proposto é ligeiramente maior que o RMS obtido pelo método baseado somente num par estereoscópico, mas, entretanto, o GSD das imagens usadas nos experimentos apresentados neste artigo é bem maior ( $1,3 \mathrm{~m}$ contra $0,25 \mathrm{~m}$ ). Portanto, é possível prever que em condições semelhantes o MDT possibilite a obtenção de resultados com maior acurácia, como, aliás, foi verificado em Dal Poz et al. (2010a).

\section{CONCLUSÕES E PERSPECTIVAS FUTURAS}

Este trabalho apresentou um método semiautomático para extração de rodovias a partir de um estereopar de imagens aéreas de baixa resolução e de um MDT, tendo por base a otimização por PD no espaço-objeto. O MDT permite restringir a busca pela linha poligonal ótima ao longo de uma faixa estreita sobre ele. Permite também eliminar linhas poligonais candidatas que não atendam ao critério de suavidade vertical, correspondendo àquelas que o ângulo de deflexão vertical supera, em um ou mais vértices, um limiar pré-definido por um operador.

A fim de avaliar o desempenho do método, foram realizados experimentos com base em dois estereopares de imagens aéreas de baixa resolução e em um MDT com resolução de $1 \mathrm{~m}$. Quarenta trechos de rodovia foram extraídos, dos quais 4 $(10 \%)$ com diferentes características foram selecionados e detalhadamente analisados. Os resultados obtidos foram analisados de forma qualitativa $\mathrm{e}$ quantitativa, sendo esta última forma baseada nos índices de completeza e correção. Em geral, pode-se notar visualmente que as linhas poligonais extraídas são geometricamente de boa qualidade, embora o parâmetro de correção para um dos casos analisados ficou um pouco abaixo de 70\%. Como mostrou um dos exemplos, quando a rodovia possui forma suave e é bem contrastada com as regiões adjacentes, mesmo que pequenas anomalias estejam presentes, o resultado obtido é aceitável. A falta de contraste num dos lados (por exemplo, provocada por solo exposto) ou em ambos os lados (por exemplo, devido à obstrução quase que total de vegetação) pode ser controlada com o posicionamento correto de pontos sementes e com a restrição de suavidade incorporada no modelo de rodovia. O exemplo envolvendo o trecho 4 mostrou que seções de rodovia de curva sinuosa podem ser controladas com a colocação adequada de apenas três pontos sementes. Os valores de RMS obtidos variaram no intervalo [1,25 m; 2,12 m] e guardaram relação direta com a largura da rodovia.

Uma das recomendações para trabalho futuro é a extensão do método para tratar imagens de alta resolução $(\mathrm{GSD}<0,7 \mathrm{~m})$, bem como MDTs de altíssima 
resolução. Um MDT com estas qualidades permite diferenciar as texturas do leito de rolagem da rodovia e das regiões adjacentes, possibilitando, por exemplo, a extração de longos trechos curvos de rodovia totalmente obstruídos. Outra direção para trabalhos futuros compreende o desenvolvimento de aplicações explorando as características do método, como a atualização e refinamento geométrico de base de dados de rodovia.

\section{AGRADECIMENTOS}

Agradeço ao Departamento de Matemática da Unemat, Campus Sinop, por viabilizar a realização do mestrado que originou os resultados desta pesquisa. Agradeço ao Programa de Pós-Graduação em Ciências Cartográficas da Unesp, Campus Presidente Prudente, por oportunizar uma convivência intensa com um ambiente de pesquisa disciplinado, sadio e acolhedor.

\section{REFERÊNCIAS BIBLIOGRÁFICAS}

AGOURIS, P.; GYFTAKIS, S.; STEFANIDIS, A. Uncertainty in image-based change detection. In: accuracy 2000, Proceedings... Amsterdã, p. 1-8, 2000.

BAJCSY, R.; TAVAKOLI, M. Computer recognition of roads from satellite picture. IEEE Transactions on Systems, Man and Cybernetics, v. 6, p. 76-84, 1976.

BALlARD, D. H.; BROWN, C. M. Computer Vision. Englewood Cliffs, New Jersey: Prentice Hall, 523 p., 1982.

BAUMGARTNER, A.; STEGER, C.; MAYER, H.; ECKSTEIN, W.; EBNER, H. Automatic Road Extraction Based on Multi-Scale, Grouping and Context. Photogrammetric Engineering and Remote Sensing 65(7). pp. 777-785, 1999.

DAL POZ, A. P.; GALLIS, R. B. A.; SILVA, J. F. C. Three-Dimensional Semiautomatic Road Extraction from a High-Resolution Aerial Image by Dynamic Programming Optimization in the Object-Space. IEEE Geoscience and Remote Sensing Letters (Print), v. 7, p. 796-800, 2010a.

DAL POZ, A. P.; GALLIS, R. B. A.; SILVA, J. F.C. Extração semi-automática de rodovia baseada em um par estéreo de imagens aéreas e em otimização por programação dinâmica no espaço-objeto. Boletim de Ciências Geodésicas, v. 16, p. 189-209, 2010 b.

GALLIS, R. B. A. Extração semi-automática da malha viária em imagens aéreas digitais de áreas rurais utilizando otimização por programação dinâmica no espaço objeto. 2006. 167 f. Tese (Ciências Cartográficas), Faculdade de Ciência e Tecnologia, Presidente Prudente.

GEMAEL, C. Introdução ao Ajustamento de Observações - aplicações Geodésicas. Editora UFPr, 1994.

GÖPFERT, J.; ROTTENSTEINER, F.; HEIPKE, C. Using snakes for the registration of topographic road database objects to ALS features. ISPRS Journal of Phrotogrammetry and Remote Sensing, v. 66, pp. 858-871, 2011. 
GRUEN, A.; LI, H. Semi-automatic linear feature extraction by dynamic programming and LSB-snakes. Photogrammetric Engineering and Remote Sensing, v. 63, n. 8, pp 985-995, 1997.

HINZ, S.; BAUMGARTNER, A.; MAYER, H.; WIEDEMANN, C.; EBNER, H. Road Extraction Focussing on Urban Areas. In: Automatic extraction of manmade objects from aerial and space images, Proceedings...Rotterdam, p. $255-$ 265, 2001.

HINZ S.; BAUMGARTNER A. Automatic Extraction of Urban Road Networks from Multi-View Aerial Imagery. ISPRS Journal of Photogrammetry and Remote Sensing, 58/1-2, pp. 83 - 98, 2003.

HINZ S.; WIEDEMANN C. Increasing Efficiency of Road Extraction by SelfDiagnosis. ISPRS Journal of Photogrammetry and Remote Sensing, 70(12), pp. 979-986, 2004.

HU, X.; TAO, C.V.; HU, Y. Automatic Road Extraction from Dense Urban Area by Integrated Processing of High Resolution Imagery and Lidar Data. In: XXth ISPRS Congress, Istanbul, Turkey, 2004, (CD-ROM).

HU, J.; RAZDAN, A.; FEMIANE, J. C.; CUI, M.; WONKA, P. Road network extraction and intersection detection from aerial images by tracking road footprints. IEEE Transaction on Geosciences and Remote Sensing, 50(12), pp. 4144-4157, 2007.

KASS, M., WITKIN, A., TERZOPOULOS, D. Snakes: Active Contour Models. In: 1st International Conference on Computer Vision, London, England: 259-268, 1987.

KIM, T.; PARK, S-R.; KIM, M-G.; JUNG, S.; KIM, K-O. Tracking road centerlines from high resolution remote sensing images by least squares correlation matching. Photogrammetric Engineering and Remote Sensing , v. 70, n. 12, pp. 1417-1422, 2004.

KIRTHIKA A.; MOOKAMBIGA A. Automated Road Network Extraction Using Artificial Neural Network. In: IEEE-International Conference on Recent Trends in Information Technology, pp. 1061-1065, 2011.

LIN, X; ZHANG, J.; LIU Z.; SHEN, J. Integration method of profile matching and template matching for road extraction from high resolution remotely sensed imagery. In: International Workshop on Earth Observation and Remote Sensing Applications, 2008, CD-ROM.

MAIA, J. L. Metodologia para avaliação de rodovias extraídas computacionalmente em imagens digitais. 2003. 116 f. Dissertação (Mestrado em Ciências Cartográficas) - FCT/Universidade Estadual Paulista, Presidente Prudente.

MAKAROVIK, B. Digital Mono-Ploters. I.T.C. Journal, Vol. 1 p. 101-122, 1973.

MARTINS, E.F.O., Extração semi-automática de rodovias no espaço-objeto: uso integrado de um estéreo par de imagens aéreas e um MDT. 2010. $97 \mathrm{f}$. Dissertação (Mestrado em Ciências Cartográficas) - FCT/Universidade Estadual Paulista, Presidente Prudente. 
MCKEOWN, D. M., DENLINGER, J. L., Cooperative methods for road tracking in aerial imagery. In: Workshop of Comp. Vision and Pattern Recogn., p. 662672, 1988.

MERLET, N.; ZERUBIA, J. New prospects in line detection by dynamic programming. IEEE Transaction on Pattern Analysis and Machine Intelligence, v. 18, n. 4, p. 426-431, 1996.

NEUENSCHWADER, W. M.; FUA, P.; IVERSON, L.; SZEKELY, G.; KUBLER, O. Ziplock snakes. International Journal of Computer Vision, v. 25, n. 6, p. 191-201, 1997.

POULLIS, C.; YOU, S. Delineation and geometric modeling of road networks. ISPRS Journal of Phrotogrammetry and Remote Sensing, v. 65, pp. 165-181, 2010.

QUAM, A. Road tracking and anomaly detection in aerial imagery. In: DARPA Image Understanding Workshop, p. 51-55, 1978.

WIEDEMANN, C.; HINZ, S. Automatic extraction and evaluation of road networks form satellite imagery. In: International Archives or Photogrammetry and remote Sensing, Vol. 32, CD-ROM, 1999.

YANG, H.; KE-JU, K. Road Extraction from Remote Sensing Imagery Based on Road Tracking and Ribbon Snake. In: Pacific-Asia Conference on Knowledge Engineering and Software Engineering, pp. 201-204, 2009.

YIBO; L. LILI,X; HUI, P. Semi-automatic road extraction from high-resolution remote sensing image: Review and prospects. In: 9th International Conference on Hybrid Intelligent System, pp. 204-209, 2009.

ZHANG, C. Towards an operational system for automated updating of road databases by integration of imagery and geodata. ISPRS Journal of Photogrammetry and Remote Sensing 58(3-4), pp.166-186, 2004.

ZHOU, J.; BISCHOF, W. F.; CAELLI, T. Road tracking in aerial images based on human - computer interaction and Bayesian filtering. ISPRS Journal of Photogrammetry and Remote Sensing 61(1), pp.108- 124, 2006.

(Recebido em junho 2011. Aceito em agosto de 2012.) 